\title{
Immunogenicity and safety of subunit influenza vaccines in pregnant women
}

\author{
Mikhail P. Kostinov ${ }^{1,2}$, Alexander P. Cherdantsev³, Nelli K. Akhmatova ${ }^{1}$, \\ Daria A. Praulova ${ }^{4}$, Aristitsa M. Kostinova ${ }^{5}$, Elina A. Akhmatova ${ }^{2}$ and \\ Evgeniia 0. Demina ${ }^{1}$
}

Affiliations: ${ }^{1}$ Mechnikov Research Institute of Vaccines and Sera, Moscow, Russia. ${ }^{2}$ Sechenov First Moscow State Medical University, Moscow, Russia. ${ }^{3}$ Federal State Budget-funded Educational Establishment of Higher Professional Education Ulyanovsk State University, Ulyanovsk, Russia. ${ }^{4}$ Federal State Budget-funded Establishment "Federal Research Clinical Center of Pediatric Hematology, Oncology and Immunology named after Dmitriy Rogachev" of the Russian Federation Ministry of Health, Moscow, Russia. ${ }^{5}$ National Research Center - Institute of Immunology Federal Medical-Biological Agency of Russia, Moscow, Russia.

Correspondence: Nelli K. Akhmatova, Mechnikov Research Institute of Vaccines and Sera, M. Kazennyi per. 5a, Moscow, 105064, Russia. E-mail: anelly555dagmail.com

ABSTRACT Pregnancy is a condition of modulated immune suppression, so this group of patients has increased risk of infectious diseases.

Trivalent subunit vaccines, unadjusted Agrippal S1 (group I) and immunoadjuvant Grippol Plus (group II), containing $5 \mu \mathrm{g}$ of actual influenza virus strains, were administered respectively to 37 and 42 women in the second and third trimester of physiological pregnancy.

The administration of subunit influenza vaccines was accompanied by the development of local reactions in no more than $10 \%$ of patients, compared with $4.9 \%$ of the 41 pregnant women in the placebo group (group III). Systemic reactions were of a general somatic nature, did not differ between vaccinated and placebo groups, and were not associated with vaccination. Physiological births in groups I, II and III were $94.6 \%, 92.9 \%$ and $85.4 \%$, respectively, and the birth rates of children without pathologies were $91.9 \%$, $90.5 \%$ and $80.5 \%$, respectively, and were comparable between groups. Vaccination stimulated the production of protective antibodies against influenza virus strains in $64.8-94.5 \%$ of patients after immunisation with an unadjusted vaccine and in $72.5-90.0 \%$ of patients after the administration of an immunoadjuvant vaccine. After 9 months, antibody levels were recorded in $51.3-72.9 \%$ in group I and $54.2-74.2 \%$ in group II. Immunisation against influenza in pregnant women provided a high level of seroprotection and seroconversion. Nevertheless, the level of seroprotection against the influenza strain $\mathrm{A}(\mathrm{H} 3 \mathrm{~N} 2$, Victoria) was slightly lower in the group immunised with an unadjusted vaccine compared to those vaccinated with the immunoadjuvant vaccine.

@ERSpublications

The trivalent subunit anti-influenza vaccines Agrippal S1 and Grippol Plus are an effective means of preventing influenza for pregnant women http://ow.ly/fCJS30iFvfz

Cite this article as: Kostinov MP, Cherdantsev AP, Akhmatova NK, et al. Immunogenicity and safety of subunit influenza vaccines in pregnant women. ERJ Open Res 2018; 4: 00060-2017 [https:// doi.org/10.1183/23120541.00060-2017].

Received: May 082017 | Accepted after revision: Feb 082018

Copyright $\odot$ ERS 2018. This article is open access and distributed under the terms of the Creative Commons Attribution Non-Commercial Licence 4.0. 


\section{Introduction}

In medical practice, immunogenicity of influenza vaccines is more apparent than their safety, although both characteristics are indicative of their epidemiological and clinical safety. Pregnancy is a condition of modulated immune suppression; therefore, this group of patients has increased risk of infectious diseases [1]. The low vaccination rates of pregnant women officially recommended in the Russian Federation since 2009 necessitate further investigation of the efficacy of influenza vaccine prophylaxis in this patient population. There have been several studies that have demonstrated the safety of vaccination during the second and third trimesters of pregnancy, and even during the first trimester, in the presence of risks without any attributable adverse fetal, perinatal or infant outcomes [2], particularly in women with a complicated premorbid background (especially asthma and diabetes) [3-7]. A combination of cardiac, respiratory, hormonal and immunological changes accompany pregnancy, which impair responses to infection and increase the likelihood of serious complications requiring hospitalisation $[8,9]$. Furthermore, several large-scale studies have reported an inverse relationship between maternal vaccination and stillbirth (risk ratios $0.56,0.66,0.59-0.74,0.77$ and 0.79 ) [10-14], although only some of these inverse relationships reached statistical significance. The mechanisms that increase the risk of serious complications from influenza during pregnancy are incompletely understood.

Whereas the safety of vaccination has been evaluated in almost all studies, its immunogenicity is assessed significantly less frequently, but studies that have directly compared adjuvant and non-adjuvanted vaccines are insufficient [15-18]. Furthermore, it is known that immunological responses generated by influenza immunisation are comparable in pregnant and non-pregnant women and can provide protection to the fetus and infant by transferring specific antibodies across the placenta [19] and in breast milk [20]. Data are insufficient with regard to the scale of antibody production in pregnant women following administration of adjuvant-containing subunit vaccines. This is particularly important, because one of these polymeric-subunit vaccines takes priority in the Russian Federation, being one of the first adjuvant vaccines worldwide to be recommended for pregnant women [21].

The goal of this study was to perform a comparative immunogenicity analysis of different subunit influenza vaccines in pregnant women.

\section{Methods}

\section{Study design and population}

This study was a prospective, randomised, open-label, comparative, parallel-group trial in pregnant women conducted in accordance with the approved Protocol of the Russian Federation National Standard, SISS R 52379-2005 "Good Clinical Practice", and the international Good Clinical Practice guidelines [22]. The study was also in agreement with the ethical norms and recommendations of the World Health Organization (WHO) and the Ministry of Health and Social Development of the Russian Federation, as well as study protocols approved by the Scientific Council of the Federal State Budget-funded Establishment Scientific Research Institute of Vaccines and Sera named after I.I. Mechnikov of the Russian Academy of Medical Sciences (Moscow) and the Ethics Committee of the Ulyanovsk State University (Protocol No. 2 from January 18, 2010) [21, 23].

\section{Data sources}

Patients were recruited in the Ulyanovsk region of the Russian Federation from August 2010 to March 2012. Signed informed consent was received from all participants. Groups of pregnant women that participated in the clinical study were formed during the outpatient medical check-up of women during primary and repeated (planned) consultation by obstetrician-gynaecologists. Selection of candidates for vaccination was carried out in the maternity welfare centre and perinatal centre in Ulyanovsk, as well as in the central district hospitals of Mayn and Tereng in the Ulyanovsk region. Vaccination was carried out at the local place of obstetric-gynaecological monitoring of the pregnant woman.

\section{Inclusion criteria}

Inclusion criteria were as follows. 1) Healthy pregnant women aged 20-40 years. 2) Volunteers capable of fulfilling the protocol requirements (i.e. able to fill in the self-observation diary and turn up for the scheduled visits). 3) Signed informed consent of the volunteers to participate in the clinical study.

\section{Exclusion criteria}

Exclusion criteria were as follows. 1) History of leukaemia, oncological conditions or positive tests for HIV, hepatitis B and C. 2) Volunteers who had received immunoglobulin preparations or blood transfusions within the last 3 months prior to the study. 3) Long-term ( $>14$ days) administration of immunodepressants or other immunomodulating drugs within the last 6 months prior to the study. 4) Any confirmed or suspected immunosuppressive or immunodeficiency disorder. 5) History of chronic 
alcohol abuse and/or substance abuse. 6) Presence of respiratory or cardiovascular insufficiency, or hepatic or renal impairment, revealed during physical examination or by laboratory tests at visit 1. 7) Severe congenital defects or serious chronic diseases including any clinically significant diseases of the lungs, kidneys, cardiovascular system or nervous system, psychiatric diseases or metabolic disorders confirmed by anamnestic data or objective clinical examination. 8) Presence of acute infectious and/or non-infectious diseases at the time of enrolment in the study. 9) Women who had influenza or influenza-like illnesses, or vaccination against influenza, within 1 year before the onset of pregnancy. 10) Pregnancy via an in vitro fertilisation procedure.

\section{Clinical characteristics of observation groups}

We followed up 37 pregnant women administered the Agrippal S1 non-adjuvanted vaccine (Novartis Vaccines and Diagnostics, Siena, Italy) (group I), as well as 42 pregnant women immunised with the trivalent inactivated polymeric-subunit preparation Grippol Plus (adjuvanted vaccine) (NPO Petrovax Pharm LLC, Moscow, Russia) (group II). Vaccination was given as a single injection in weeks 16-30 of a physiological pregnancy. To assess the clinical safety of vaccination, a third group of pregnant women was selected to receive a placebo (group III). As a placebo, we used phosphate-buffered saline (GlaxoSmithKline Biologicals, Rixensart, Belgium), which is used as a diluent for lyophilised vaccines and was administered once. The mean \pm SD ages of groups I, II and III were $27.8 \pm 0.6,23.3 \pm 0.4$ and $24.1 \pm 0.3$ years, respectively. When assessing the general state of the women's health, we were guided by current and anamnestic data on infectious and somatic diseases, and obstetric history. The clinical characteristics of the comparison groups are shown in table 1 . The analysis of the premorbid conditions diagnosed in enrolled pregnant subjects yielded rather high levels of hypochromic anaemia (45\%), thyroid disorders (21-27\%), vegetative dysfunction (a Russian diagnosis for the group of mild undiagnosed neurological diseases such as panic attack, depression or anxiety disorders) (23-24\%), threatening spontaneous abortion (32-35\%), and active forms of chronic genitourinary infections verified by PCR (35\%). There were no significant differences in initial clinical status between the observed groups.

Monitoring of pregnant women before and after the vaccination was carried out integrally with obstetrician-gynaecologists in accordance with the requirements of the Order of the Ministry of Health and Social Development of the Russian Federation No. 808n of 02.10.2009 "On the Approval of the Procedure for Obtaining Obstetric-Gynecological Care". Before the vaccination, after obtaining signed informed consent from the woman to participate in the clinical trial, a general laboratory examination was conducted, including blood sampling for general analysis, biochemical parameters and a general urine test. In the absence of deviations from the normative values, a pregnant woman was invited to receive immunisation against influenza.

\section{Clinical safety assessment}

Clinical safety of vaccination was assessed by analysis of the development of local and systemic reactions in the early (0-7 days) and late (8-30 days) periods after vaccine administration. In addition, the characteristics of pregnancy and delivery outcomes were given.

\section{Serological methods of research and terms of their conduct}

Venous blood samples were tested in pregnancy weeks 16-30 before immunisation, and at 1 month, 3 months, 5-6 months (post partum days 2-3) and 8-9 months (post partum day 90) after immunisation. Antibodies to influenza A and B viruses were detected in the haemagglutination inhibition assay using

\section{TABLE 1 Background conditions observed in pregnant study subjects immunised against} influenza

\begin{tabular}{lccc} 
Background condition & $\begin{array}{c}\text { Group I } \\
\text { Agrippal S1 }\end{array}$ & $\begin{array}{c}\text { Group II } \\
\text { Grippol Plus }\end{array}$ & $\begin{array}{c}\text { Group III } \\
\text { Placebo }\end{array}$ \\
\hline Total subjects & 37 & 42 & 41 \\
Anaemia of pregnancy & $17(45.9)$ & $19(45.2)$ & $25(60.9)$ \\
Chronic genitourinary infections & $13(35.1)$ & $15(35.7)$ & $17(41.5)$ \\
Thyroid disorders & $10(27.0)$ & $9(21.4)$ & $12(29.3)$ \\
Neuro-circulatory dystonia syndrome & $9(24.3)$ & $10(23.8)$ & $12(29.3)$ \\
Threatening miscarriage & $13(35.1)$ & $12(32.4)$ & $21(51.2)$ \\
\hline
\end{tabular}


$0.75 \%$ cock erythrocyte suspension following preliminary heating of the tested sera to $56^{\circ} \mathrm{C}$ for $1 \mathrm{~h}$ (to remove nonspecific inhibitors and improve assay sensitivity). The antigens used included the vaccine strains A(H1N1, California 7, 2009), A(H3N2, Victoria) and B(Brisbane).

\section{Vaccine preparations}

The difference between the studied vaccines was in the presence of the water-soluble high-molecular-weight immunoadjuvant Polyoxidonium included in the Grippol Plus vaccine (500 mg per $0.5 \mathrm{~mL}$, which is one immunising dose), which allowed a three-fold reduction in the content of influenza virus $\mathrm{H}$-antigens (to $5 \mathrm{mg}$ ) compared with the imported reference non-adjuvanted vaccine. The antigenic composition of the administered products met the WHO and European Union recommendations for a 2010-2011 and 2011-2012 seasonal vaccine. The strain composition of all influenza vaccines was the same in those seasons.

Polyoxidonium is well established as an adjuvant in different in vivo and in vitro studies, especially among patients with immune suppressed conditions [24]. Different studies have demonstrated good tolerability of adjuvanted vaccine and no effect on fetus or child development [25-27].

\section{Definitions}

Since no conventional immunogenicity criteria are currently available for a vaccine for pregnant women and for patients at high risk for development of severe influenza infection forms, we employed the standard efficacy criteria for adult patients recommended by the Committee for Proprietary Medicinal Products (CPMP), which are as follows. 1) The seroprotection level (the proportion of immunised patients with haemagglutinin inhibiting antibody $(\mathrm{HIAB})$ titres $\geqslant 1: 40$ by post-vaccination day 21 ) must be $\geqslant 70 \%$. 2) The seroconversion level or immunologically active vaccine (the relative number of immunised patients with HIAB titres increasing more than four-fold compared with baseline for all individuals achieving immunoprotection) must be $\geqslant 40 \%$. 3) The seroconversion factor or geometric mean increase (elevation of geometric mean HIAB titres on day 21 compared with baseline) must be at least 2.5 -fold. A vaccine is considered immunogenic and effective if it meets at least one of the three listed criteria. Pregnant women with HIAB titres over 1:20 but lower than 1:40 were viewed as seropositive (vaccine responders with low specific immunity levels).

\section{Statistical analysis}

Obtained data were analysed using descriptive statistical methods, following preliminary use of Thompson's rule. Significance of differences in quantitative parameters for obtained data was calculated using Wilcoxon's nonparametric test for independent samples. The AtteStat10 statistical application package was utilised. The seroconversion factor was determined by obtaining the logarithm for the geometric mean of a data set (Microsoft Excel 2003).

\section{Results}

\section{Clinical safety of vaccination}

The clinical course of the early post-vaccination period in pregnant women vaccinated against influenza is reflected in table 2. It should be noted that the difference between the vaccinated and placebo groups in the development of local reactions was for reactions that did not require the prescription of any medication and disappeared on their own after several days, without causing disruption of the patient's activity. The incidence of systemic reactions in the first week after vaccination was higher than that of local reactions, but was comparable between vaccinated groups and did not exceed the values obtained in the placebo group. The appearance of a cough or sore throat in all groups of patients characterised the appearance of a respiratory infection.

The late post-vaccination period in pregnant women was characterised mainly by the registration of systemic reactions, without significant differences among the observed groups (table 3). At the same time, the probability of occurrence of such symptoms as fatigue, headache, dizziness and abdominal pain prevailed over other common symptoms that characterise the well-being of pregnant women.

\section{Pregnancy outcomes}

In most cases in the observation groups (85.4-94.6\%), the pregnancy ended with physiological birth (table 4). In some women the pregnancy ended prematurely, with the birth of premature babies (2.7-9.8\%). Cases of the birth of children with neurological pathology of the perinatal period, mainly associated with gestational immaturity, were also described (7.3-9.5\%). The syndrome of intrauterine infection was registered (2.7-7.3\%), as were anomalies and malformations (2.4-4.9\%). 


\begin{tabular}{|c|c|c|c|}
\hline Clinical sign & $\begin{array}{c}\text { Group I } \\
\text { Agrippal S1 }\end{array}$ & $\begin{array}{c}\text { Group II } \\
\text { Grippol Plus }\end{array}$ & $\begin{array}{l}\text { Group III } \\
\text { Placebo }\end{array}$ \\
\hline Total subjects & 37 & 42 & 41 \\
\hline \multicolumn{4}{|l|}{ Local reactions } \\
\hline Pain at the injection site & $3(8.1)^{*}$ & $2(4.8)^{*}$ & $1(2.4)$ \\
\hline Hyperaemia at the injection site & $4(10.8)^{*}$ & $4(9.5)^{*}$ & $0(0.0)$ \\
\hline Sealing at the injection site & $3(8.1)^{*}$ & 3 (7.1)* & $1(2.4)$ \\
\hline \multicolumn{4}{|l|}{ Systemic reactions } \\
\hline Enhanced temperature & $1(2.7)$ & $2(4.8)$ & $1(2.4)$ \\
\hline Fatigability & $10(27.0)$ & $11(26.2)$ & 12 (29.3) \\
\hline Arthralgia & $2(5.4)$ & $2(4.8)$ & $2(4.9)$ \\
\hline Myalgia & $3(8.1)$ & $2(4.8)$ & $3(7.3)$ \\
\hline Headache & $10(27.0)$ & $11(26.2)$ & 8 (19.5) \\
\hline Dizziness & $3(8.1)$ & 5 (11.9) & $5(12.2)$ \\
\hline Nausea & $4(10.8)$ & $8(19.0)$ & 5 (12.2) \\
\hline Stomach ache & $3(8.1)$ & 5 (11.9) & $6(14.6)$ \\
\hline Diarrhoea & $1(2.7)$ & $1(2.4)$ & $1(2.4)$ \\
\hline \multicolumn{4}{|l|}{ Infection } \\
\hline Cough & $0(0.0)$ & $0(0.0)$ & $1(2.4)$ \\
\hline Pain in the throat & $1(2.7)$ & $2(4.8)$ & $2(4.9)$ \\
\hline
\end{tabular}

Data are presented as $n$ or $n(\%) . *$ : $p<0.05$ between the compared vaccinated and placebo groups. Note that $p>0.05$ for the differences between the compared vaccinated groups.

\section{Post-vaccination immunity}

The baseline and pre-immunisation anti-influenza A virus antibody concentrations were found to be the same in the pregnant subjects (table 5). It should be mentioned that prior to influenza immunisation, we detected some pregnant women who had rather high baseline antibody levels $(\geqslant 1: 40)$, ensuring efficient protection from probable development of infection. In particular, the proportions of patients achieving seroprotection were found to be $16.2 \%$ and $9.5 \%$ for seroprotection against the $\mathrm{A}(\mathrm{H} 1 \mathrm{~N} 1)$ strains in groups I and II, respectively $(\mathrm{p}<0.05) ; 18.9 \%$ and $19.0 \%$ against the $\mathrm{A}(\mathrm{H} 3 \mathrm{~N} 2)$ strains in groups I and II, respectively; and $48.7 \%$ and $23.8 \%$ against the $\mathrm{B}$ (Brisbane) strain in groups I and II, respectively $(\mathrm{p}<0.01)$.

\section{TABLE 3 Clinical course of the late (8-30 days) post-vaccination period in pregnant women}

\begin{tabular}{lccc} 
Clinical sign & $\begin{array}{c}\text { Group I } \\
\text { Agrippal S1 }\end{array}$ & $\begin{array}{c}\text { Group II } \\
\text { Grippol Plus }\end{array}$ & $\begin{array}{c}\text { Group III } \\
\text { Placebo }\end{array}$ \\
\hline $\begin{array}{l}\text { Total subjects } \\
\text { Local reactions }\end{array}$ & 37 & 42 & 41 \\
$\quad$ Pain at the injection site & $0(0.0)$ & $0(0.0)$ & $0(0.0)$ \\
Hyperaemia at the injection site & $0(0.0)$ & $0(0.0)$ & $0(0.0)$ \\
Sealing at the injection site & $0(0.0)$ & $0(0.0)$ & $0(0.0)$ \\
Systemic reactions & $2(5.4)$ & $4(9.5)$ & $4(9.8)$ \\
Enhanced temperature & $10(27.0)$ & $8(19.0)$ & $7(17.1)$ \\
Fatigability & $2(5.4)$ & $2(4.8)$ & $1(2.4)$ \\
Arthralgia & $2(5.4)$ & $3(7.1)$ & $1(2.4)$ \\
Myalgia & $12(32.4)$ & $5(23.8)$ & $10(24.4)$ \\
Headache & $4(10.8)$ & $8(1.9)$ & $6(14.6)$ \\
Dizziness & $5(13.5)$ & $7(16.7)$ & $8(19.5)$ \\
Nausea & $7(18.9)$ & $4(9.5)$ & $6(14.6)$ \\
Stomach ache & $3(8.1)$ & & $3(7.3)$ \\
Diarrhoea & & $7(16.7)$ & $7(17.1)$ \\
Infection & $4(10.8)$ & $8(19.0)$ & $7(17.1)$ \\
Cough & $8(21.6)$ & &
\end{tabular}

Data are presented as $n$ or $n(\%)$. Note that $p>0.05$ for the differences between the compared groups. 


\begin{tabular}{|c|c|c|c|}
\hline & $\begin{array}{c}\text { Group I } \\
\text { Agrippal S1 }\end{array}$ & $\begin{array}{c}\text { Group II } \\
\text { Grippol Plus }\end{array}$ & $\begin{array}{l}\text { Group III } \\
\text { Placebo }\end{array}$ \\
\hline Total subjects & 37 & 42 & 41 \\
\hline \multicolumn{4}{|l|}{ Women } \\
\hline Physiological birth & $35(94.6)$ & 39 (92.9) & $35(85.4)$ \\
\hline Miscarriage & $1(2.7)$ & $1(2.4)$ & $2(4.9)$ \\
\hline Premature birth & $1(2.7)$ & $2(4.8)$ & $4(9.8)$ \\
\hline \multicolumn{4}{|l|}{ Children } \\
\hline Without pathology & 34 (91.9) & 38 (90.5) & 33 (80.5) \\
\hline With abnormalities or malformations & $1(2.7)$ & $1(2.4)$ & $2(4.9)$ \\
\hline Perinatal affection of CNS & $3(8.1)$ & $4(9.5)$ & $3(7.3)$ \\
\hline Intrauterine infection syndrome & $1(2.7)$ & $2(4.8)$ & $3(7.3)$ \\
\hline
\end{tabular}

Data are presented as $n$ or $n(\%)$. Note that $p>0.05$ for the differences between the compared groups. CNS: central nervous system.

The geometric mean titre in the compared groups was below the conditionally protective level for antibodies to the influenza A virus strains and higher than that level for antibodies to the influenza B virus strain, being $22.9 \pm 0.2$ in group I and $14.8 \pm 0.3$ in group II $(\mathrm{p}<0.05)$. At 1 month and at 3 months post immunisation, the proportions of pregnant women seropositive to all influenza virus strains were found to be significantly increased regardless of the study arm (table 5). Differences were observed in the number of women seropositive to the $\mathrm{A}(\mathrm{H} 3 \mathrm{~N} 2)$ strain at 5-6 months post immunisation (i.e. on the very first postnatal days) $(62.1 \%$ for group I and $78.3 \%$ for group II) $(\mathrm{p}<0.05)$ and at $8-9$ months after vaccination (at 3 months post partum) $(51.3 \%$ for group I and $71.4 \%$ for group II) $(p<0.05)$.

Seroprotection against the influenza $\mathrm{A}$ and $\mathrm{B}$ virus strains intensifies significantly during the post-immunisation period, retaining almost the same values at 8-9 months after administration of either

\section{TABLE 5 Post-immunisation immunity to influenza virus strains in pregnant study subjects}

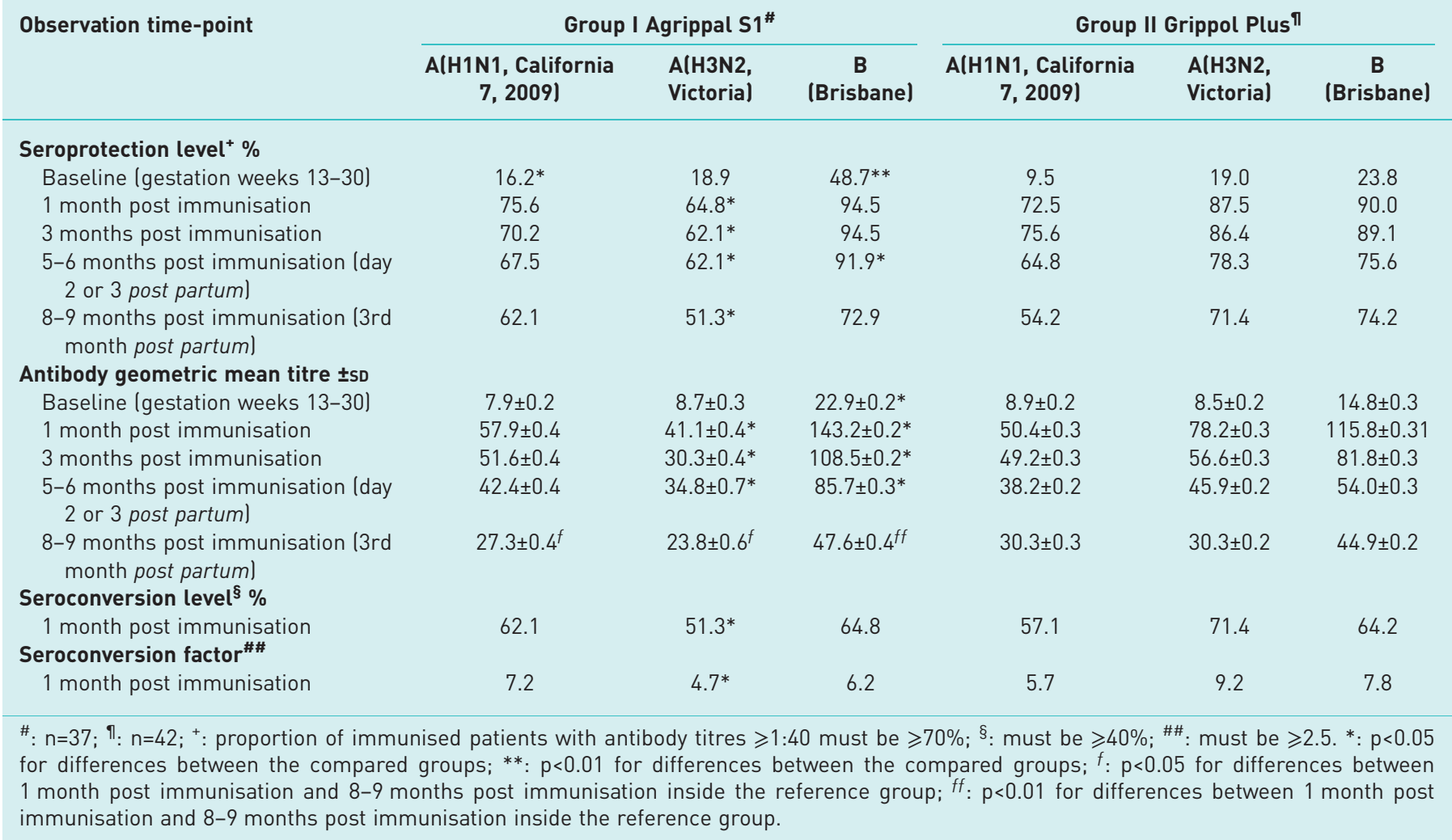


of the vaccine preparations. Nevertheless, pregnant women administered non-adjuvanted vaccine had lower anti-A(H3N2) seroprotection levels at 1 month, 3 months and $8-9$ months, as compared with the group receiving the adjuvanted vaccine.

Increased geometric mean titres of antibodies to all influenza virus strains were observed in the post-immunisation period in pregnant subjects. Pregnant women immunised with non-adjuvanted vaccine had lower geometric mean titres for antibodies to the $\mathrm{A}(\mathrm{H} 3 \mathrm{~N} 2)$ strain at 1 month, 3 months and 5-6 months post immunisation than those in subjects immunised with adjuvanted vaccine; however, the geometric mean titres for antibodies to the influenza B strain registered at the same time-points ( 1 month, 3 months and 5-6 months) were higher (table 5).

The seroconversion level for the analysed influenza virus strains at 1 month after immunisation was higher than $40 \%$ in both study groups (which meets the CPMP criteria), although the group I level of anti-A(H3N2) seroconversion was lower $(51.3 \%)$ than that in group II $(71.4 \%)(\mathrm{p}<0.05)$.

Among immunised pregnant women, the seroconversion factor also exceeded, more than 2.5-fold, the efficacy level recommended for vaccines, with the highest value (9.2) obtained for the $\mathrm{A}(\mathrm{H} 3 \mathrm{~N} 2)$ strain in group II (versus 4.7 in group I; $\mathrm{p}<0.05$ ).

The proportion of subjects seronegative to the evaluated influenza strains, $\mathrm{A}(\mathrm{H} 1 \mathrm{~N} 1), \mathrm{A}(\mathrm{H} 3 \mathrm{~N} 2)$ and $\mathrm{B}(\mathrm{Brisbane})$, did not exceed $21.6 \%$ for group I and $17.5 \%$ for group II during the first month post immunisation.

\section{Discussion}

Assessment of the clinical picture of pregnant women subject to vaccination, as well as placebo groups, showed the presence of concomitant somatic pathology, which does not differ from the indices of other authors [28-30]. These indicators in the post-vaccination period were not taken into consideration. Similar premorbid backgrounds were observed in the two study groups, which permitted a comparative qualitative analysis of the clinical tolerability and immunological efficacy of the influenza immunisation. There was the same high proportion of women with underlying comorbidities in both groups, but these women were not registered in any clinics as patients with chronic diseases. There were no outbreaks of these diseases during the pregnancy. If we assume that these comorbidities could influence the efficacy of vaccination, the data would be affected in both groups.

The introduction of subunit vaccines to pregnant women, regardless of the production technology, was accompanied in the first week by the development of local reactions more often than in the placebo group. At the same time, the frequency, severity and duration of these reactions did not differ between the compared vaccinated groups. However, it is important to note that these reactions were rare when the placebo phosphate-buffered saline solution was administered. The frequency of development of systemic reactions did not differ between groups of pregnant women vaccinated with unadjusted and adjuvant preparations against influenza, and these systemic reactions were often subjective in nature and their interrelation with immunisation is unlikely. In favour of this argument is their similar development after the injection that did not contain vaccine strains (placebo group) [31, 32]. Registration of cough or sore throat, regardless of the administration of vaccine preparations and water-salt solution, cannot be considered as a negative effect of vaccinations but rather as a coincidence of circumstances with the simultaneous appearance of respiratory infections of the upper respiratory tract.

The emergence of systemic reactions in the late post-vaccination period can be regarded as background symptoms accompanying the course of pregnancy. In addition, they differ little from those that occurred in the early period after immunisation. Cough or sore throat signified the appearance of respiratory infections, which proceeded in mild form; the treatment consisted mainly of local application of decongestants or irrigation of the oral cavity with antiseptic solutions, without development of complications. The described symptoms and complaints made by pregnant women were not regarded as side-effects of vaccination and did not affect the subsequent course of pregnancy.

Despite the fact that the teratogenic effect on the development of the fetus of subunit vaccines against influenza, including those containing adjuvants, has been previously studied, it was of interest to estimate the outcome of pregnancy after vaccines in the second and third trimesters. It should be noted that the physiological births in the groups administered with both vaccines met with the same frequency, not differing from the placebo group, and coincided with the frequency of this indicator recorded in the Ulyanovsk region where this study was conducted. This outcome was due to obstetric pathology, which had no link with previous vaccination. Note that the congenital anomalies and malformations among vaccinated and unvaccinated (placebo) pregnant women were the same. The frequency of anomalies and malformations among the observed groups did not differ from the mean values for the Ulyanovsk region $(3.8-5.9 \%)$. 
The baseline concentrations of antibodies to the evaluated influenza A and B virus strains demonstrated that some of the subjects had conditionally protective titres defining the seropositive state, as evidence of a previous infection, although it was not approved by documentation and collected anamnesis of patients. The obtained results demonstrate a prolonged circulation of this virus resulting in annual epidemic outbreaks [33]. Since in each group there was almost the same number of pregnant women with initially protective antibodies to strains of the influenza A and B viruses, and also due to their small number, it was considered inappropriate to separate them into separate subgroups.

Despite the differences in the concentrations of initially protective levels of antibodies to influenza $\mathrm{A}(\mathrm{H} 1 \mathrm{~N} 1)$ and $\mathrm{B}$ virus strains in the observed groups, after immunisation the growth of specific antibodies to all vaccine strains, regardless of the drug administered, was noted. It should be emphasised that pregnant women who received an adjuvant vaccine had antibody levels to the $\mathrm{A}(\mathrm{H} 3 \mathrm{~N} 2)$ influenza virus that were $22.7 \%, 24.3 \%$ and $20.1 \%$ higher at 1,3 and $8-9$ months, respectively, than subjects immunised with a non-adjuvanted vaccine. The levels of antibodies to the strain of influenza B virus were registered higher by $16.3 \%$ at 5-6 months after vaccination of pregnant women with an unadjusted vaccine, but after 8-9 months these differences were levelled. Nevertheless, by analysing the levels of protection against influenza in vaccinated pregnant women with one of the two vaccines, a fairly strong seroprotection can be observed within 8-9 months after immunisation.

The study of the average geometric antibody titres in the observation dynamics showed differences in the values of the $\mathrm{A}(\mathrm{H} 3 \mathrm{~N} 2)$ influenza virus among pregnant women vaccinated with an immunoadjuvant preparation, in which they were higher in comparison with the pregnant women receiving the unadjusted vaccine. Conversely, the average geometric titres of antibodies to the influenza B virus strain were higher at all observation times in subjects vaccinated with a conventional subunit vaccine. It is important to note that these differences disappear 8-9 months after immunisation, regardless of the vaccine used. It is interesting that there were no differences between the groups according to the average geometric titres of antibodies to the strain of the $\mathrm{A}(\mathrm{H} 1 \mathrm{~N} 1)$ virus.

Despite the fact that the levels of seroprotection, determined at 1 month and 8-9 months after immunisation, did not differ statistically, except for the $\mathrm{A}(\mathrm{H} 1 \mathrm{~N} 1)$ strain in group $\mathrm{I}$, the average geometric amount of antibodies received in 8-9 months was lower, regardless of the vaccine used, except for the $\mathrm{A}(\mathrm{H} 3 \mathrm{~N} 2)$ virus strain in group I and $\mathrm{A}(\mathrm{H} 1 \mathrm{~N} 1)$ in group II, which corresponded to the antibody level after 1 month after vaccination.

The level and seroconversion factor reflecting the increase in antibody titres after immunisation against influenza were high during the first month after vaccination in both study groups, although there were some differences (a relatively lower increase in antibody titre after the first month after immunisation against influenza $\mathrm{A}(\mathrm{H} 3 \mathrm{~N} 2)$ in group I).

Thus, the administration of subunit influenza vaccines to women in the second and third trimester of pregnancy does not lead to the development of unusual phenomena that cause a complicated course of pregnancy. In the post-vaccination period, a symptomatic complex of systemic reactions was most often associated with the psychoemotional state of pregnant women, due to the fear of the possible development of an adverse effect of vaccination. After vaccination, antibodies to all strains of the influenza virus were observed at a conventionally protective level with an increase to $64.8-94.5 \%$ after immunisation with the subunit vaccine and to $72.5-90.0 \%$ after the administration of the immunoadjuvant vaccine. Although the number of cases of seropositive pregnant women declined in the post-immunisation period, we demonstrated that the majority of subjects immunised against influenza were still protected from three strains of the influenza virus 8-9 months after vaccination (the proportions changed to $51.3-72.9 \%$ in group I and to $54.2-74.2 \%$ in group II). Another interesting result was that during the epidemic season, there were no cases of influenza among the immunised.

In conclusion, the most advanced trivalent subunit anti-influenza vaccines, Agrippal S1 and Grippol Plus, are an effective means of specifically preventing influenza for pregnant women, in full compliance with the requirements of the CPMP. The immunogenicity of subunit influenza vaccines in pregnant subjects is confirmed by the fact that 1 month after vaccination, a high level of seroprotection, seroconversion and high intensity of influenza immunity are provided. After the introduction of an immunoadjuvant vaccine with a reduced amount of antigens ( $5 \mu \mathrm{g}$ of each of the influenza virus strains), the criteria for CPMP were fully met for all strains of the influenza virus included in the vaccine.

Acknowledgements: The authors would like to thank the study participants and staff members of the different study sites for their contribution to the study.

Author contributions: M.P. Kostinov conceived and designed the study and assessed the results. A.P. Cherdantsev performed laboratory and statistical analyses and interpreted the results. N.K. Akhmatova interpreted the results. 
D.A. Praulova followed up and collected clinical information about the patients. A.M. Kostinova and E.O. Demina updated the database. All authors critically reviewed the manuscript and gave their final approval of the version for submission.

Conflict of interest: None declared.

\section{References}

1 Mor G, Cardenas I. The immune system in pregnancy: a unique complexity. Am J Reprod Immunol 2010; 63: 425-433.

2 Zaman K, Roy E, Arifeen SE, et al. Effectiveness of maternal influenza immunization in mothers and infants N Engl J Med 2008; 359: 1555-1564

3 Hartert TV, Neuzil KM, Shintani AK, et al. Maternal morbidity and perinatal outcomes among pregnant women with respiratory hospitalizations during influenza season. Am J Obstet Gynecol 2003; 189: 1705-1712.

4 Neuzil KM, Reed GW, Mitchel EF, et al. Impact of influenza on acute cardiopulmonary hospitalizations in pregnant women. Am J Epidemiol 1998; 148: 1094-1102.

5 Englund JA. Maternal immunization with inactivated influenza vaccine: rationale and experience. Vaccine 2003; 21: $3460-3464$.

6 World Health Organization. Influenza vaccines. WHO Position Paper. Weekly Epidemiological Record 2005; 80: 279-287. www.who.int/entity/wer/2005/wer8033.pdf

7 National Center for Immunization and Respiratory Diseases. Vaccination During Pregnancy. In: General recommendations on immunization - recommendations of the Advisory Committee on Immunization Practices (ACIP). MMWR Recomm Rep 2011; 60: 26-27.

8 Mighty HE. Acute respiratory failure in pregnancy. Clin Obst Gynecol 2010; 53: 360-368.

9 Centers for Disease Control and Prevention. Pregnant Women and Influenza (Flu). www.cdc.gov/flu/protect/ vaccine/pregnant.htm Date last updated: November 29, 2017. Date last accessed: March 7, 2018.

10 Fell DB, Sprague AE, Liu N, et al. H1N1 influenza vaccination during pregnancy and fetal and neonatal outcomes. Am J Public Health 2012; 102: e33-e40.

11 Pasternak B, Svanström H, Mølgaard-Nielsen D, et al. Vaccination against pandemic A/H1N1 2009 influenza in pregnancy and risk of fetal death: cohort study in Denmark. BMJ 2012; 344: e2794.

12 Beau $\mathrm{AB}$, Hurault-Delarue $\mathrm{C}$, Vidal $\mathrm{S}$, et al. Pandemic A/H1N1 influenza vaccination during pregnancy: a comparative study using the EFEMERIS database. Vaccine 2014; 32: 1254-1258.

13 Källén B, Olausson PO. Vaccination against H1N1 influenza with Pandemrix during pregnancy and delivery outcome: a Swedish register study. BJOG 2012; 119: 1583-1590.

14 Sammon CJ, Snowball J, McGrogan A, et al. Evaluating the hazard of foetal death following H1N1 influenza vaccination; a population based cohort study in the UK GPRD. PLoS One 2012; 7: e51734.

15 Madhi SA, Cutland CL, Kuwanda L. Influenza vaccination of pregnant women and protection of their infants. N Engl J Med 2014; 371: 918-931.

16 Lin SY, Wu ET, Lin CH, et al. The safety and immunogenicity of trivalent inactivated influenza vaccination: a study of maternal-cord blood pairs in Taiwan. PLoS One 2013; 8: e62983.

17 Blanchard-Rohner G, Meier S, Bel M, et al. Influenza vaccination given at least 2 weeks before delivery to pregnant women facilitates transmission of seroprotective influenza-specific antibodies to the newborn. Pediatr Infect Dis J 2013; 32: 1374-1380.

18 Mutsaerts E, Madhi SA, Cutland CL, et al. Influenza vaccination of pregnant women protects them over two consecutive influenza seasons in a randomized controlled trial. Expert Rev Vaccines 2016; 15: 1055-1062.

19 Puck JM, Glezen WP, Frank AL, et al. Protection of infants from infection with influenza A virus by transplacentally acquired antibody. J Infect Dis 1980; 142: 844-849.

20 Schlaudecker EP, Steinhoff MC, Omer SB, et al. IgA and neutralizing antibodies to influenza A virus in human milk: a randomized trial of antenatal influenza immunization. PLoS One 2013; 8: e70867.

21 Minzdravsoc. [Informational letter of the Ministry of Health and Social Development of the Russian Federation of December 11, 2009, No. 15-4/3108-07. Recommendations on the organization and conduct of vaccination of pregnant women and women in the hospital against influenza A (H1N1)]. www.garant.ru/products/ipo/prime/ doc/4089732/ Date last updated: March 2, 2010. Date last accessed: March 13, 2018.

22 Federal Agency for Technical Regulation and Metrology. [National Standard of the Russian Federation. Good Clinical Practice. ISO 52379-2005]. Moscow, 2005. http://docs.cntd.ru/document/1200041147

23 Kostinov MP, Cherdantsev AP, Shmitko AD, et al. Duration of preservation of antibodies to the flu virus in the mother-child pairs during the vaccination of women depending on the trimester of pregnancy. Int J Biomed 2015; 5: 179-183.

24 Zverev VV, Semenov BF, Khaitov RM, eds. [Vaccines and Vaccination: National Guidelines]. Moscow, GEOTAR-Media, 2011; pp. 47-55.

25 Kostinov MP, Cherdantsev AP. [The clinical and immunological safety of inactivated immunologic adjuvant influenza subunit vaccine for pregnant women]. Akusherstvo I Ginekologiya [Obstetrics and Gynecology] 2016; 2: 64-69.

26 Kostinov MP, Cherdantsev AP. [Health state of children born from pregnant women vaccinated against influenza]. Pediatria Journal named after GN Speransky 2016; 95: 67-71.

27 Kostinov MP, Cherdantsev AP, Semenova SS, et al. [Obstetric and perinatal outcomes among pregnant women after influenza vaccination and after transferred respiratory infection]. Ginekologiya [Gynecology] 2015; 17: 43-46.

28 Sidelnikova VM, Antonov AG. [Premature birth. A premature baby: A Guide for Physicians]. Moscow, GEOTAR-Media, 2006; pp. 116-127.

29 Syundyukova EG, Medvedev BI, Sachenkov SL, et al. The value of somatic and obstetric-gynecologic pathology in the development of preeclampsia. Bulletin of the South Ural State University. Series: Education, Health, Physical Culture, 2013; 13: 102-107.

30 Koul PA, Bali NK, Mir H, et al. Influenza illness in pregnant Indian women: a cross-sectional study. Infect Dis Obstet Gynecol 2016; 2016: 1248470 
31 Kostinov MP, Cherdantsev AP, Savisko AA, et al. True and false reactions in pregnant women to introduction of influenza vaccine. Gynecol Obstet Perinatol 2011; 10: 38-42.

32 Kostinov MP, Cherdantsev AP, Shmitko AD, et al. The efficacy of immunoadjuvant-containing influenza vaccines in pregnancy. In: Afrin F, Hemeg H, Ozbak H, eds. Vaccines. London, InTechOpen, 2017; pp. 67-94.

33 Ministry of Health of the Russian Federation. Report on the health of the population and the organization of public health services on the basis of the performance of executive authorities of the constituent entities of the Russian Federation (2014). Available from: www.rosminzdrav.ru/ministry/programms/doklad-o-sostoyanii-zdorovyanaseleniya-i-organizatsii-zdravoohraneniya-po-itogam-deyatelnosti-organov-ispolnitelnoy-vlasti-sub-ektov-rossiyskoyfederatsii-za-2014-god Date last updated: June 18, 2015. Date last accessed: March 13, 2018. 